Tugrul A. Aktash', Gunel N. Aslanova ${ }^{2}$

DOI: 10.25045/jpit.v07.i1.10

${ }^{1}$ University of Yalova, Yalova, Turkey

${ }^{2}$ Institute of Information Technology of ANAS, Baku, Azerbaijan

1'taktas@yalova.edu.tr ${ }^{2}$ gunel_aslanova90@mail.ru

\title{
RECOGNITION OF PERSON BASED ON VASCULAR PATTERNS AND DEVELOPMENT OF RECOGNITION SYSTEM
}

Recognition of person, based on his/her vascular patterns, is one of the newest methods of the biometric recognition systems. Many studies have recently been conducted in this field. The article proposes solution for a compelling factor in vascular recognition system. The research is focused on solving the problem of reduced quality of recognition due to the swelling of persons vessels when the hand of a person is exposed to load. As a results of carrying heavy loads for long periods, the veins of the hand of a person are swollen. In this case, problems in hand vein recognition may arise. In this article, the method is proposed for solving these problems.

Keywords: Vein Recognition, Identification, Authentication, Vascular Pattern, Vascular Network.

\section{Introduction}

Biometric technologies use human physiological parameters (fingerprints, iris of the eye's retina etc.) and behavior to realize identification of a person. The application of these technologies plays an important role in preventing terrorism, arms and drug trafficking, and illegal migrations. Biometric recognition, which is of great importance in our lives, is one of the most promising methods for reliable identification [1]. In modern times, the recognition, based on the handvascular patterns of a person, is widely used at airports, security systems, for the use of credit cards and so on. By exploring the biometric features of an individual, the biometric system compares them to the biometric data stored in data-base. These systems are used in the information security systems, therefore, they must be very sensitive [2].

At present, biometric recognition is widely used due to the rapidly increased importance of information security systems. Hand-vein recognition systems are used in many fields. For example, many Japanese banks began to use these systems since July 2004, to verify the identity of customers. Fujitsu is going to apply this technology in various areas by reducing the size of the sensors and increasing the control speed of this technology [3].

\section{Available methods for the vascular pattern recognition of a person}

Currently, some systems achieve the recognition with the thin vascular network of a finger of a person. Nevertheless, very complex network of the thin veins decreases the quality of the recognition of an individual. Some systems carry out the recognition of a person according to the vascular network of the palm. Over the last decade, the studies on the palm-vein recognition systems have gradually grown. The working principle of such recognition systems is as follows: the receiver (sensor) reveals the traces of the blood vessels by sending them infrared rays. The absorption of infrared rays by hemoglobin in the blood reveals these traces. The recognition is more promising due to the hemoglobin used here and the presence of blood in veins. The advantage of this system is the fact that, in case of hand cuts or damage, the structure of the vessels does not alter or affect the system's performance [4]. Figure 1 shows the vascular network of the right hand [5].

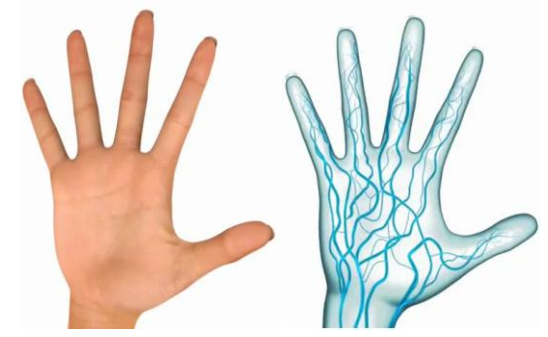

Figure 1. Description of the vascular network of the right hand 
Acquisition of the description of the vascular network of a human hand is a simpler procedure than the description acquisition of other biometric systems. A camera with an infrared lighting may achieve the description in approximately $5-15 \mathrm{~cm}$ distance.

First, a hand is placed into the device and prepared by placing an item between the fingers [6].

The working principle of the system is as follows: hemoglobin in the blood partially absorbs infrared rays emitted from a series of LEDs (light-emitting diode) placed side-by-side passed through the hand. The areas of ray absorption (i.e. the blood vessels of hand) appear as dark regions in the image taken by camera, placed on the other side of hand [7].

\section{Developing hand-vein description acquisition system}

A recognition system is developed by the authors to conduct an experiment. The system set up is as follows. First, the digital camera needs to be infrared light-sensitive. As shown in Figure 2, Nikon D80 is equipped with a filter screening the infrared light [8]. This filter is placed behind the lens, in front of the main motherboard inside the camera.

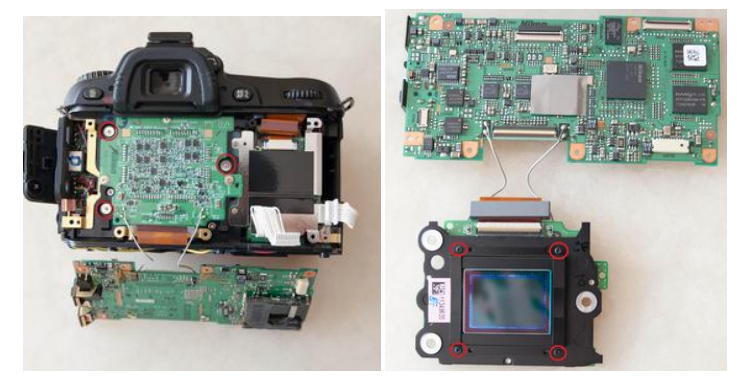

Figure 2. Nikon D80 camera

Removing the filter allows the infrared rays to enter the camera. However, in order to enable only the visible light to enter the camera, low conductance filter needs to be placed. IR Low Pass Filter, brought by researcher from the United States, was added to the camera.

380-780 nm wavelength interval of light does not pass through the center of the filter Central wave length of this filter does not exceed the 380-780 nm interval of light [9]. Changing the filter is not sufficient. Light source entering the camera should also feature lights that are in infrared wave interval

The spectrum of light emitted by the sun is illustrated in Figure 3 [10]. As you can see from the image, the greater spectrum of light, infrared light has a very small volume. Infrared lights are devided: short, medium and long wave lengths. The selected filter functions at shorter wavelengths. Therefore, the light source is provided by means of LEDs.

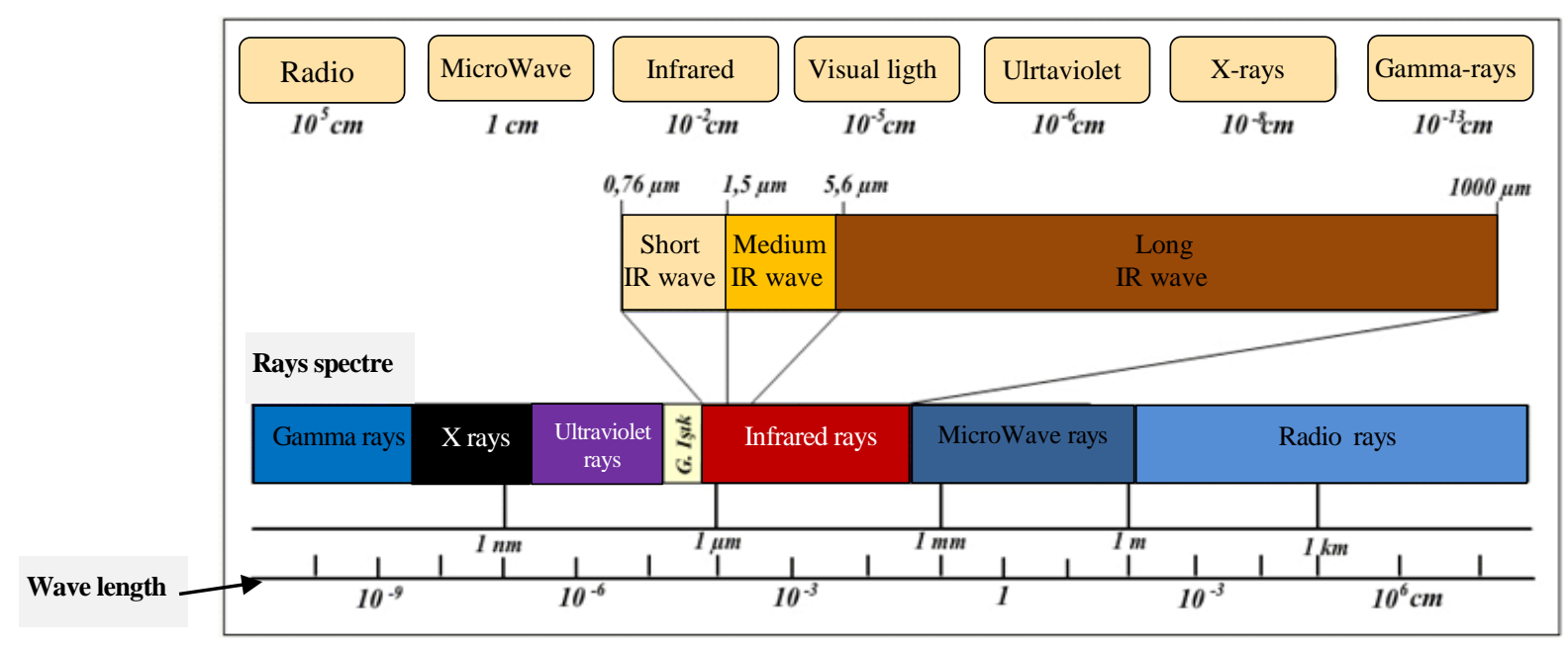

Figure 3. The spectrum of the light system 
In this regard, the LED is available in 3 types: $720 \mathrm{~nm}, 850 \mathrm{~nm}$ and $940 \mathrm{~nm}$. As the purchased filter cuts the $720 \mathrm{~nm}$ wavelength, this LED should not be used. VSLY5850 and TSAL6200 LEDs are selected. Two different sources are developed with either LEDs, and one LED, which performs the best result, is picked for preparing the system device.

LEDs are the light falling on the severity of the strain to be stable on the need to stabilize the movement. In this case, the LEDs current and voltage regulator circuit is activated, you need to add one. Figure 4 as a light source in the investigation has been developed [11]. In Figure 5, the electrical and electronics equipment needed for the establishment of the system are described.

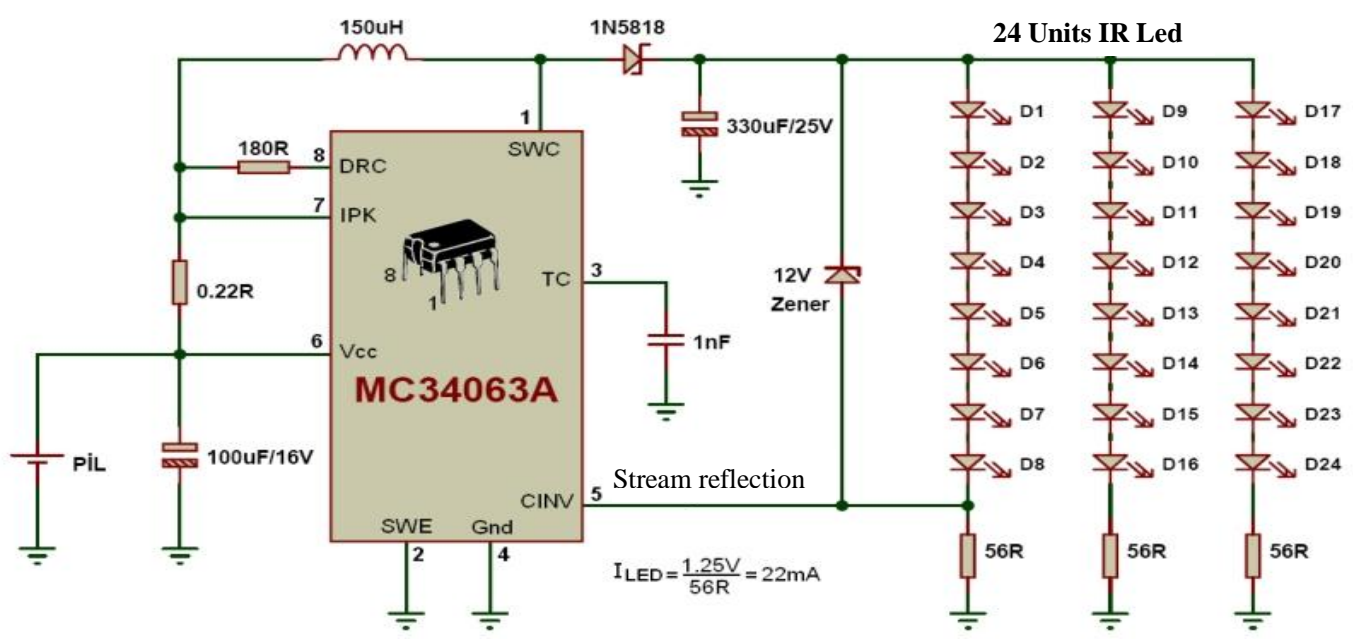

Figure 4. The light source system

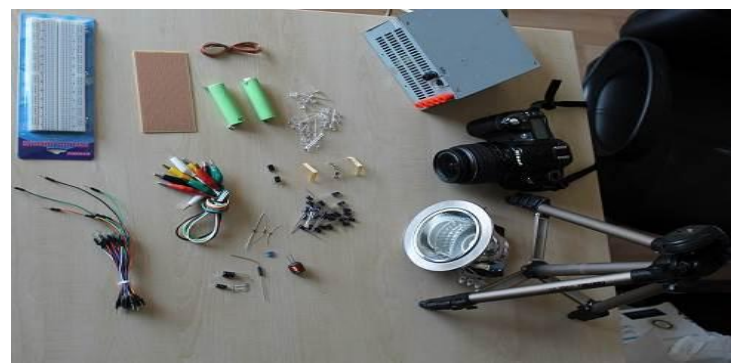

Figure 5. The need for the establishment of the system of electrical and electronic equipment

Tripod is to mounted to the $30 * 50 \mathrm{~cm}$ thin board. Then, the light source and the camera need to be fixed on tripod.Two nails, allowing placement of hand, should be put on the panel. In this manner, the recognition system is being developed.

First, hand needs to located in the mechanism with the fashion that nails fit between the fingers. This system is described in Figure 6. Figure 7 shows the side view of the system [12]. Person recognition process is implemented by applying two different methods to obtained vascular patterns.

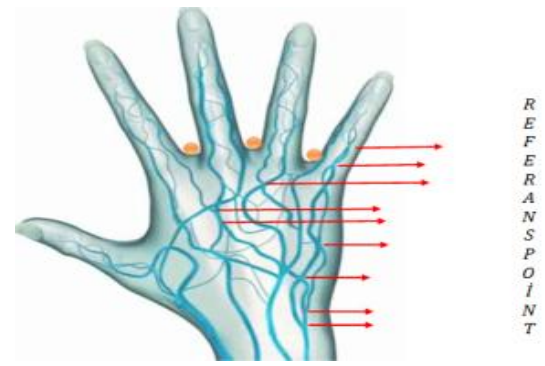

Figure 6. Specific points in the vessels of hand 


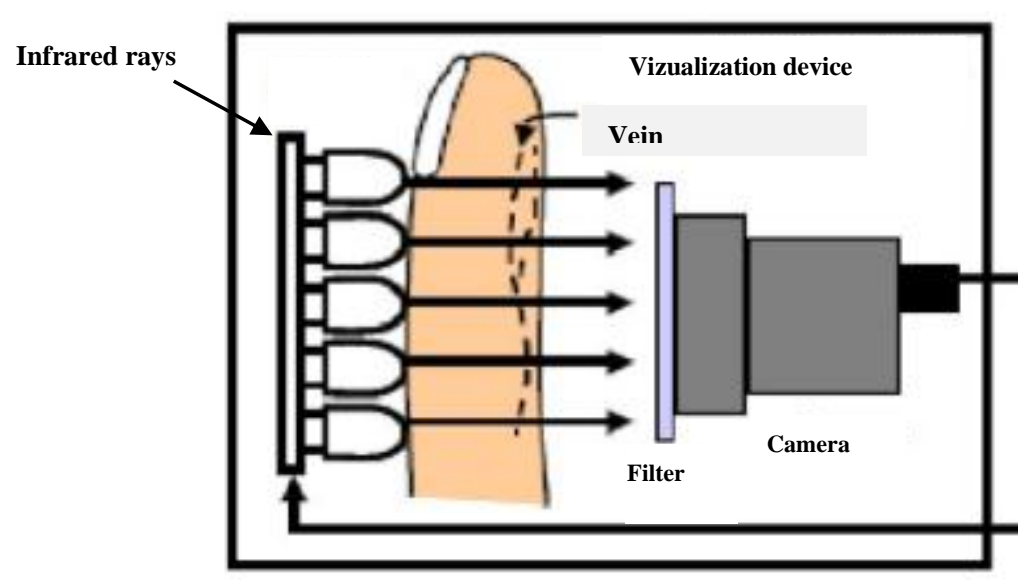

Figure 7. Vein recognition system

In the first method, the image, first, is converted into the 0-255 color range (gray scale). Then, the value and the value point is a point of great prices for white color, black and white imagery is converted into black color at low prices. Then, value point is determined and the image is converted into black and white by converting high values into white and low values into black colors. As seen in Figure 8, the reference points are taken on the basis of one piece of hand image. A grey image, consisting of vascular patterns, is obtained. In the next stage, the image is fixed and digitalized the lines located on the vessel.

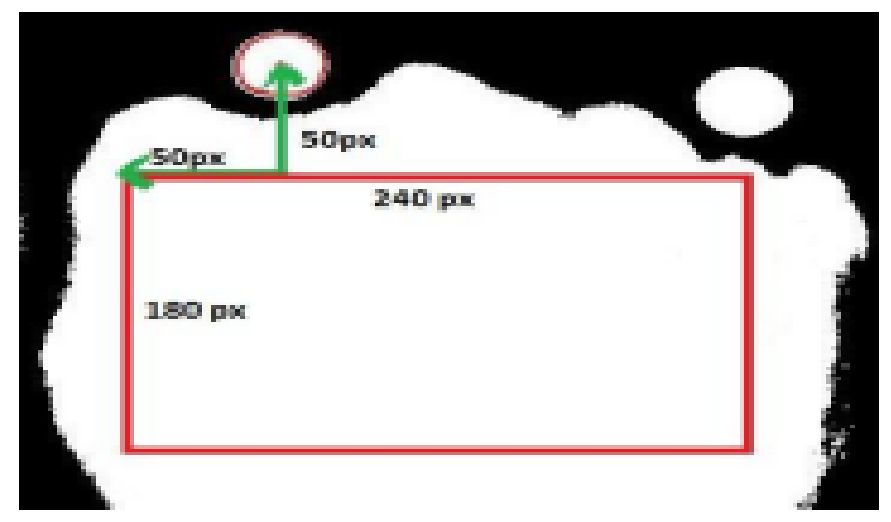

Figure 8. A piece derived from hand image

With the purpose of cancelling additional parts (hair, skin etc.), filtering method is applied. Then, operations such as histogram equalization is carried out to provide noticeability of the vascular structure. Histogram curve gathered around the set with a ton of synchronization histogram (0-255) in the correct tones are distributed in a way that the appearance of the image is provided in a homogeneous distribution of gray tones. The vascular structure of a pattern of black and white to become the Otsu algorithm was used [13]. This type of pattern issues, with the aim of reaching an average absolute deviation method of digital data is used. The average absolute deviation of the vector used to obtain a specific method for each pixel in the image, calculating the cost of the operation is carried out in deviation from the average price of pixels [14]. After this stage, each sub-making process described in the 20x20 size matrices into the vein pattern in black and white in the 01 encoded form. This database is created with matrices. Later, the system comparison between the patterns read by the system and the patterns stored in recognition database is performed. 
The second method will be applied in a similar fingerprint recognition [15]. Border region where the picture was photographed hand in hand, only to find the algorithm found. Then the level of gray scale is being reduced and color normalization is performed. Wiener and Median filters are applied to the image to obtain more noticeable vascular structure after normalization [16].

After this operation, derive of vascular patterns is realized through utilization of boundary determination method. Since all borders exist in the image, additional filter is used to strain the image from noise by determining certain edges. Thus, pure vein patterns map is obtained [17]. For example, a vein pattern is shown in Figure 9.

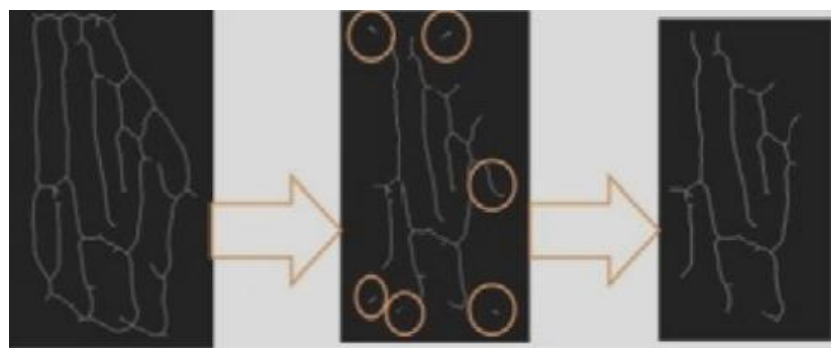

Figure 9. The vein pattern map

Splitting and final dots derived from this image are calculated and stored in a database in the form of a matrix. Recognition operation is implemented by equalizing fingerprints with the images stored in the database.

\section{Problem Statement}

Shape of blood vessels are subject to changes under some circumstances: some people gain or lose weight because of illness, pregnancy or excessive eating. In addition to weight gain, swelling of the hands, especially in pregnant women is observed. In the case if swellngs and weight gains and loss are extreme, changes occur also to the hand veins. It can cause problems in the process of recognition [18-21].

Blood vessels get swollen as a result of increased blood pressure, when a person carries heavy loads for long periods. In this case, the hand veins recognition of the problem may occur. Traces of blood vessel are typically taken at room temperature. However, those living in colder climates, especially if cold weather for a long time, the contraction will occur in the veins of the hands. In this case, the blood vessels in the hand-recognition system is experiencing problems. At the same time thermal (heat) because of the machine outside the room temperature is very cold environment, such as thermal cameras can not be described. Image quality affects quality of recognition. When body floats in the sea for a long time, hand of a person gets shrunk.. It is due water in the cells, which comes out through diffusion. Shrunk hand is a little different from normal blood trail. In this case, effectiveness rate of a system recognizing the clients of a hotel by the seaside on the bases of vascular patterns falls down

In recognition of this research through the veins of the hand, the hand of a person is exposed to cargo vessels, swelling in the hand because of the recognition is aimed at solving the problem of reducing quality. There are very few studies on the subject [1-3]. This technique has been used in studies of the device differently. But, no one has been able to overcome the technical problem completely. Hand vascular changes difficult to track down the reasons for the widespread use of systems to find a solution to this type of recognition is of great importance.

\section{Problem solving with algorithm}

The research was conducted with 100 people sample population. Four images of vascular patterns of the hand were taken from each person at different time, under room temperature, throughout daily life. Moreover, image of vascular pattern of the hand was taken before training and every 30 
minute throughout total 2 hour training in the gym. Totally, 7 images were taken from each person. Figure 10 illustrates images of two different people of hand veins before and after training [21].

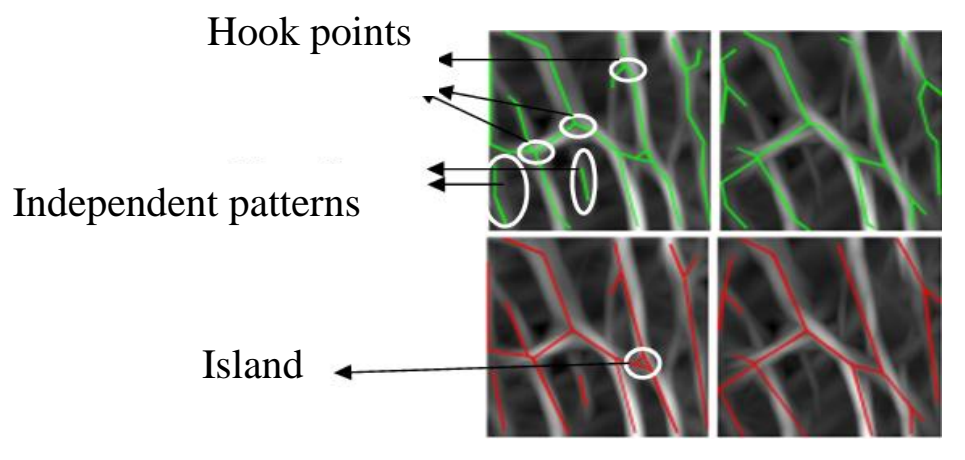

Figure 10. Hand images of two people before and after training.

Hand prints vessels are examined and it is observed that curves in veins disappear after sport. The device is converted into a matrix followed by studies in general, vascular tracks the score of 1 , giving the data is stored in other parts 0 score. Later, recognition is carried out by comparison matrices. But as seen in the pictures above, the vein recognition systems are changing and difficult part of the trail vein recognition factor when it comes down to quality.

The article applies a different method. If we pay attention to the signs of hand veins, there are splittings, islands and independent areas in palm veinas well. In the first person, there are 9 splitting points, 2 independent areas and 1 island. Although there are 7 splitting points in the second person, no independent areas or island were determined. These three points are considered as basis and hand vascular patterns are divided into categories according to numbers.

Comparisons will not be performed according with all hand vascular patterns stored in database, but with relevant areas only. This method will allow the system to operate more quickly, but both were fewer compared to the material, facilitate us to get the correct result.

The dots are defined as follows:

I Island. If the data is 01 indoor field in the matrix, it is considered to be an island.

Splitting II. Horizontal and vertical matrix prices 11 neighbor of at least two other cells, this is considered a splitting point.

Independent third line. Lines or a line splitting the island in the group do not exist, it is considered an independent line. Figure 11 shows on the matrix of dots.

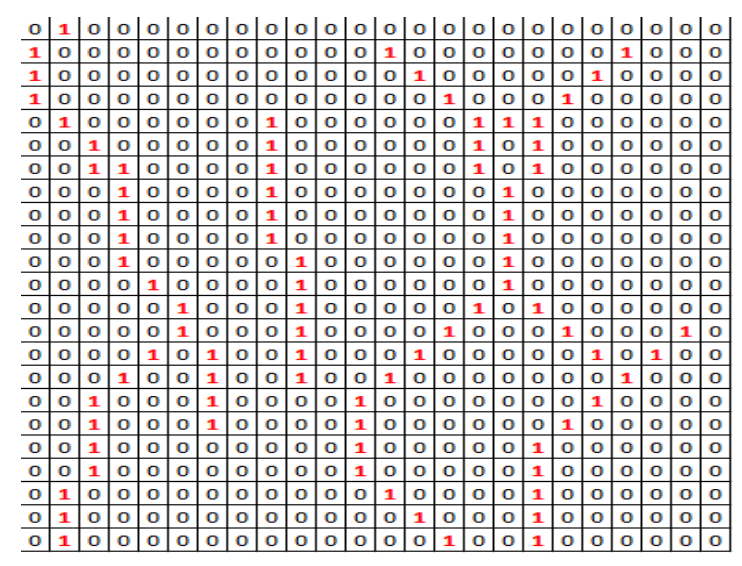

Figure 11. The matrix of vascular net

Categorizing the veins cannot solely yield accurate results. The following steps should be taken to reach the right conclusion. For example, less change in shape was observed in vein of the 
first study group home (large) size smaller than the arteries or blood vessels, which are relatively thin. However, splitting points are less affected areas in the veins. The closest distance between the dots can be saved in a matrix. After spending power could be due to a change in a similar vein, establishing a correlation matrix can be stored in a database, which is included in the score.

\section{Conclusion}

The vein recognition is one of the methods of biometric recognition in identity recognition systems. In the past 10 years, studies have been conducted on person recognition system based on thin vessels of fingers. Studies on the blood vessels of the hand is still very new. Particularly, quick weight loss or gain in a short period, working with hand for long time and exposure to cold and water changes the shape of the vessels.

In this study, data was collected from a sample population. Since the data was gathered under natural conditions, it can take a long time. Hand veins can partially be changed as the heart pumps more blood to vessels when hand is over-employed. During the study, it was observed that splitting points are impacted to lesser extent. Building correlation between these points and adapting categories on vascular patterns of hand underpins realization of recognition with better quality. While categorizing data gathered from limited number of research sample, only the island numbers will be prioritized.

The exact number of all data collected known sample group, along with the number of points splitting the vessel name, number and the number of independent lines of the group, the number of combinations can be found.

\section{Literature}

1. Shan S. Finger vein recognition technology, Master Thesis, electronic and computer training. Institute of Science and Technology, Firat University, Elazig, 2013, pp. 29-31.

2. R. Chellappa, Wilson CL, Sirohey S. Human and machine recognition of faces: A survey / Proceedings of the IEEE, 1995, vol83, No.5, pp. 705-740.

3. With the hand vascular pattern recognition methods Frugal H. ARTIFICIAL intelligence. Master Thesis, Electrical and Electronics Engineering, Selcuk University, Institute of Science, Konya, 2011, pp. 22-26.

4. http://www.ergosis.com.tr/avucici-damar-tanima-teknolojisi.html

5. http://www.pdksrehberi.com/el-damar-tanima-ile-personel-devam-kontrol-sistemi-pdks/

6. http://www.nanobiz.com.tr/images/products/mobiyosys\%20brosuru.pdf

7. Miura N., A. Nagasaka, Miyatake T. Feature extraction of finger-vein patterns based on personal identification // Repeated line tracking and its application to Machine Vision and Applications, 2004, vol15, No.4, pp. 194-203.

8. http://www.infradream.com/?p=798

9. http://www.tradeindia.com/fp254876/Infrared-850-940nm-IR-Filters.html

10. http://www.alfsatis.com/alf_infrared_teknoloji.html

11. http://www.biltek.tubitak.gov.tr/gelisim/elektronik/dosyalar/35/devre.jpg

12. L. Ma, Y. Wang and Tan T. Gabor Iris recognition based on Multichannel Filtering / The 5th Asian Conference on Computer Vision, 2002, pp. 279-283.

13. M. Soni, Phalguni M., Robust Vein Pattern Recognition System //-based Journal of Computers, 2012, vol.7, no.11, pp. 2711-2718.

14. Lakshmi DC, Kandaswamy A. An Algorithm for Improved Accuracy of fusion through the Multiple Feature Sets Unimodal Biometric Systems // ICGST-GVIP Journal, 2009, vo19, no.3, pp. 33-40.

15. Maltoni D., D. Maio, Jain, A., Prabhakar S. Handbook of Fingerprint Recognition. Springer Science \& Business Media, 2009, 494 c.

16. Rafael Ç.G., Richard EW Digital Image Processing. Tom Robbins, 2002, p. 190. 
17. L. Wang, Leedham C.G. A Thermal Hand Vein Pattern Verification System / Proc. Of the 3rd International conferenceon Advences's Pattern Recognition (ICAPR 2005), Lecture Notes in Computer Science, vol3687 // Springer, 2005, Part 2, pp. 58-63.

18. M. Soni, Gupta S, Rao MS, Gupta P. A New Vein Pattern-based Verification System // International Journal of Computer Science and Information Security, 2010, vol8, no.1, pp. 58-63.

19. L. Wang, Leedham Ç.G. Near and Far-infrared Vein Pattern for Imaging Biometrics / Proc of the IEEE International Conference on Advanced Video and Signal Based Surveillance (AVSS06), 2006, pp.58-63.

20. http://www.lafsozluk.com/2011/03/el-ile-ilgili-deyimler-ve-anlamlari.html

21. Scott P. Hand Geometry Based Vein Biometry, Computer Engineering Graduate Program in Computer Engineering // Bahcesehir University, Istanbul, 2012, pp. 58-66. 